\title{
Rezension: Fallgewichtung an schweizerischen Gerichten
}

\section{Methodik von Studien der gewichteten Geschäftslast, Grundlagen - Auslegeordnung - Folgerungen}

Ein Fall ist nicht gleich ein Fall, wenn es um das Bewältigen und Verteilen der Geschäftslast geht - das ist die Erfahrung aller daran Beteiligten. Die Unterschiede in der Beanspruchung durch verschiedene Fälle in verschiedenen Rechtsgebieten werden, so der Ausgangspunkt der hier besprochenen Arbeit, am besten mit einer Gewichtung der Fälle erfasst. Dafür gibt es verschiedene methodische Ansätze, darunter auch solche, die sich praktisch bewährt haben.

Beitragsart: Literature

Zitiervorschlag: Hans-Jakob Mosimann, Rezension: Fallgewichtung an schweizerischen Gerichten, in: «Justice - Justiz - Giustizia» 2020/4 
[1] Die Thematik der hier besprochenen Berner Dissertation ist für die Organisation, den Betrieb und die Führung eines Gerichts von eminenter praktischer Bedeutung, geht es doch um den bestmöglichen Umgang mit der Geschäftslast und deren Bewältigung. Die Arbeit behandelt in drei Hauptteilen die theoretisch-konzeptionellen Grundlagen, von Gerichten in ausgewählten Ländern schon verwendete Methoden und sich daraus ergebende Folgerungen für Gerichte in der Schweiz.

[2] Ausgangspunkt der theoretisch-konzeptionellen Überlegungen im ersten Hauptteil ist die Erkenntnis, dass reine Fallzahlen für den Umgang mit der Geschäftslast nur bedingt geeignet sind, weil verschiedene Fallarten ganz unterschiedlich viel Zeit zur Bearbeitung in Anspruch nehmen (Rz 32 ff.). Deshalb empfiehlt es sich, anhand der pro Fallkategorie durchschnittlich erforderlichen Bearbeitungszeit Fallgewichte zu bilden ( $\mathrm{Rz} 40 \mathrm{ff}$.), dies nebst der Erfassung von nichtfallbezogenen Beanspruchungen (Rz 47 ff.). Auf der Grundlage der dementsprechend gewichteten Geschäftslast kann der personelle Ressourcenbedarf ermittelt werden, und es können sowohl die Ressourcen als auch die Geschäfte verteilt werden (Rz 129 ff.). Hinsichtlich der Methodik von gewichteten Geschäftslastsstudien (Rz 147 ff.) lassen sich zwei hauptsächliche Ansätze unterscheiden, nämlich Berechnungsverfahren (Rz $154 \mathrm{ff}$.) vorzugsweise mit Zeiterhebung durch Selbstaufschreibung (Rz $163 \mathrm{ff}$.) einerseits und andererseits Schätzverfahren (Rz $168 \mathrm{ff}$.) mittels ein- oder mehrmaliger Befragung involvierter Personen.

[3] Im zweiten Hauptteil werden bereits durchgeführte Geschäftslaststudien und bewirtschaftungssysteme vorgestellt, was ein überraschend vielfältiges Panorama ergibt und hinsichtlich der Beispiele aus der Schweiz auch davon profitiert, dass die Autorin mit ausgewählten Auskunftspersonen (S. 383 ff.) Interviews durchgeführt hat. In der ersten bekannten Schweizer Studie wurden 2008 die Juristinnen und Juristen aller Verwaltungs- und Sozialversicherungsgerichte befragt (Rz 200 ff.). Weitere Studien wurden 2011 am Bundesverwaltungsgericht (Rz 217 ff.) und 2015 den Gerichten und der Staatsanwaltschaft Basel-Stadt (Rz 243 ff.) abgeschlossen. Sodann gibt es Praxisbeispiele der Fallgewichtung, so insbesondere am Sozialversicherungsgericht des Kantons Zürich (Rz 286 ff.), am Obergericht des Kantons Aargau (Rz 296 ff.) und für die Kreisgerichte im Kanton St. Gallen (Rz 302 ff.).

[4] In den USA sind Studien der gewichteten Geschäftslast weit verbreitet. Mit Anfängen schon in den 1960er Jahren basieren sie heute auf den vom National Center for State Courts (NCSC) entwickelten und in rund 40 Bundesstaaten verwendeten Ansätzen (Rz 323 ff.). Illustrativ sind die drei beispielhaft ausgewählten Staaten: Im einen resultierte eine Aufstockung der Gerichte ( $\mathrm{Rz} 367 \mathrm{ff}$.), im anderen eine Verkleinerung ( $\mathrm{Rz} 373 \mathrm{ff}$.) und im dritten eine neue Gerichtsbezirksgliederung (Rz 376 ff.). Das in Deutschland (Rz 396 ff.) entwickelte Personalbedarfsberechnungssystem PEBB§Y (Rz 417 ff.) dient bis heute der Ermittlung des Personalbedarfs (Rz 477 ff.). Weitere Beispiele betreffen die Niederlande (Rz 493 ff.) und die Personalanforderungsrechnung (PAR) in Österreich (Rz 513 ff.), sowie elf weitere Staaten (Rz 528 ff.). Zur Anwendung kommen teilweise Berechnungsverfahren, teilweise Schätzverfahren und teilweise eine Kombination der beiden, und die Untersuchungen erfolgen entweder fallbezogen oder - was vor allem bei langen Verfahrensdauern wertvoll sein kann - bezogen auf einzelne Verfahrensabschnitte oder -schritte (Rz 552 ff.). Als Verwendungszwecke von Fallgewichten (Rz 596 ff.) zu konstatieren sind: Ressourcenberechnung und -verteilung, Fallzuteilung, Leistungsbeurteilung, Reorganisation.

[5] Zukunftsgerichtet ist der dritte Hauptteil der Arbeit (Folgerungen für die Gerichte in der Schweiz). Einleitend benennt die Autorin die Kriterien bei der Methodenwahl (Rz 579 ff.), so etwa das mit der Studie verfolgte Ziel, den Anspruch an die Datenqualität, Aufwand und Kosten, 
die Grösse der erfassten Gerichtsbarkeit (Fallzahlen und Personalbestand), die Akzeptanz. Zu klären ist auch, ob es um Ist- oder Soll-Werte geht (Rz 603 ff.), welche Personalkategorien einbezogen werden ( $\mathrm{Rz} 616$ ff.) und wie es sich mit nicht-fallbezogenen Arbeiten verhält ( $\mathrm{Rz} 620$ f.). Als optimale Methodik empfiehlt die Autorin mit plausibler Begründung ein verfahrensschrittbezogenes Berechnungsverfahren (Rz $622 \mathrm{ff}$.), und sie nennt die folgenden Erfolgsfaktoren für die Umsetzung (Rz 659 ff.): Einsatz einer Begleitgruppe, massvolle Anzahl Fallkategorien, nicht zu detaillierte Verfahrensschritte, massvolle Anzahl nicht-fallbezogener Kategorien, Hauptpersonalkategorien, angemessene Erhebungsdauer, praktikable Erhebungsinstrumente, Akzeptanzförderung durch Information und Schulung, Gewährleistung der Anonymität, Pilotierung und Einsatz eines Helpdesks.

[6] In der Ausgabe 2020/2 von «Justice - Justiz - Giustizia» hat sich PAscal Baur mit der Frage «Über den Zahlen?» befasst. Vernünftigerweise wird man seine Frage wohl verneinen müssen, und dann ist man nicht über den Zahlen, sondern mittendrin. Genau hier setzt die vorliegende Arbeit an: Welche Zahlen dienen welchen Zielen am besten? Die Autorin erbringt auf eindrückliche Weise den Nachweis, wie viel zweckmässiger es ist, mit gewichteten Fallzahlen zu operieren, und sie zeigt auf, wie eine solche Gewichtung erfolgen kann und welche Überlegungen für welche Methodik sprechen. Ihr Ausgangspunkt ist die sorgfältige Analyse der Randbedingungen, welche der Justiz gesetzt sind, und ein besonderer Mehrwert ist ihre präzise und detaillierte Darstellung von bereits in der Praxis verwendeten Methoden. Dies alles macht die Arbeit zu einem ausgesprochen wertvollen Beitrag für alle an einer guten Justiz Interessierten.

Daniela WinKLer, Fallgewichtung an schweizerischen Gerichten: Methodik von Studien der gewichteten Geschäftslast, Grundlagen - Auslegeordnung - Folgerungen; Stämpfli Verlag, Bern 2020, XXIV + 387 Seiten, CHF 115.-, ISBN 978-3-7272-0772-3.

Hans-Jаков Mosimann, Dr. iur., Präsident des Sozialversicherungsgerichts des Kantons Zürich und Mitglied des Redaktionsteams der Schweizer Richterzeitung. 